\title{
Nonlinear Landau damping of nonlocal MHD waves: Exact solutions and particle acceleration
}

\author{
I. Lerche $\mathrm{L}^{1,2}$ and R. Schlickeiser ${ }^{1}$ \\ 1 Institut für Theoretische Physik, Lehrstuhl IV: Weltraum- und Astrophysik, Ruhr-Universität Bochum, \\ 44780 Bochum, Germany \\ 2 Permanent address: Department of Geological Sciences, University of South Carolina, \\ Columbia, SC 29208, USA \\ e-mail: black@geol.sc.edu
}

Received 16 July 2001 / Accepted 27 November 2001

\begin{abstract}
In order to describe correctly the heating rate of the interstellar medium by plasma wave energy losses, we investigate the influence of non-linear amplitude effects on wave characteristics, using the Rogister-Mjølhus-Wyller equation, which is the most complete plasma kinetic description for nonlinear magnetohydrodynamic (MHD) waves propagating parallel to an ambient magnetic field. It is demonstrated (1) that any energy change arises only as a consequence of the nonlocal term in this equation, and (2) that the energy transfer rate can be evaluated in terms of quadratures dependent on the initial and/or boundary conditions imposed on exact solutions to the derivative non-linear Schrödinger equation. Moreover, the Rogister-Mjølhus-Wyller equation implies the presence of oscillatory solutions, independent of the strength of the nonlocal term, indicating that such waves play a role in the long-term evolution of the interstellar medium. These waves propagate in both positive and negative senses (depending on their wavenumbers), suggesting a bifurcation of energy flux directions at large and small spatial scales. Such a division is of significance not only for the long term behavior of the interstellar medium, but also for particle energization.
\end{abstract}

Key words. ISM: magnetic fields - plasmas - waves - turbulence - magnetohydrodynamics (MHD)

\section{Introduction}

Understanding the nature of interstellar plasma turbulence at scales much larger than the ion-skin length is crucial for the dynamics of cosmic ray particles, collisionless heat transfer to the interstellar medium, and the interpretation of radio wave propagation and scintillation. From the plasma wave viewpoint, plasma irregularities are usually modeled as a superposition of waves from linear theory, such as Alfven and magnetosonic waves. The coupling between these waves, due to weak nonlinearities, gives rise to phenomena such as cascading in wavenumber space that account for the observed power law power spectra of interstellar density fluctuations.

When investigating the nature of interstellar turbulence, it is necessary to keep in mind that the interstellar medium contains a number of plasmas (commonly referred to as phases) of very diverse characteristics (Spangler 1999). The five major phases are summarized in Table 1. The observed turbulence properties as obtained from radio propagation measurements as dispersion measures, rotation measures and interstellar scintillation are biased

Send offprint requests to: R. Schlickeiser, e-mail: r.schlickeiser@tp4.ruhr-uni-bochum.de towards the high-density ionized interstellar phases with large volume filling factors, i.e. the diffuse intercloud gas and HII envelopes. Especially, dispersion measure and scintillation data are primarily diagnostics of density, and only secondarily of magnetic field. Both diagnostics indicate the existence of interstellar density irregularities with frequency power spectra extending over 11 decades in frequency, much below $\left(\omega \ll \Omega_{\mathrm{p}}\right)$ the proton gyrofrequency (Armstrong et al. 1995).

The plasma wave models allow consideration of the damping processes of the turbulence that is important for the heating of the interstellar medium (Lerche \& Schlickeiser 2001). In this context, in order to describe correctly wave energy loss in the interstellar medium, it is necessary to investigate the influence of non-linear amplitude effects on wave characteristics. Estimates of small amplitude wave energy loss rates are radically different in spatial and temporal behaviour from estimates pertaining to large amplitude waves (Mjølhus \& Wyller 1986, 1988; Spangler 1990, 1991; Dawson \& Fontan 1990; Flå et al. 1989). Here we investigate this problem on the basis of the Rogister-Mjølhus-Wyller equation, which, to date, is the most complete plasma kinetic description for nonlinear magnetohydrodynamic (MHD) waves propagating 
Table 1. Major phases of the interstellar medium.

\begin{tabular}{|l|l|r|r|}
\hline Phase & density $\left(\mathrm{cm}^{-3}\right)$ & temperature $(\mathrm{K})$ & volume filling factor $(\%)$ \\
\hline molecular cloud & $\geq 10^{4}$ & $\leq 70$ & $\leq 2$ \\
cold neutral medium & $10-100$ & $\simeq 100$ & $\leq 2$ \\
HII envelopes & $5-10$ & 8000 & $\leq 2$ \\
diffuse intercloud gas & $0.1-0.5$ & 8000 & $\simeq 50$ \\
coronal & $10^{-3}$ & $10^{6}$ & $20-80$ \\
\hline
\end{tabular}

parallel to an ambient magnetic field (Rogister 1971; Mjølhus \& Wyller 1986, 1988). With some minor modifications to the notation presented in Spangler (1991), the wave evolution is described through

$$
\begin{aligned}
& \imath \frac{\partial \phi}{\partial \tau}+\frac{\imath}{2} \frac{\partial}{\partial \xi}\left(\phi \left[N_{1}|\phi|^{2}\right.\right. \\
& \left.\left.+N_{2} \mathrm{P} \int_{-\infty}^{\infty} \frac{\mathrm{d} \xi_{0}\left|\phi\left(\xi_{0}\right)\right|^{2}}{\left(\xi_{0}-\xi\right)}\right]\right)-\mu \frac{\partial^{2} \phi}{\partial \xi^{2}}=0
\end{aligned}
$$

which reduces to the Derivative Nonlinear Schrödinger equation (DNLS) for $N_{2}=0$. The definition of symbols in Eq. (1) is as follows: $\phi=\left(b_{x}+\imath b_{y}\right) / B_{0}$, where the ambient magnetic field, $B_{0}$, is directed in the $z$-direction, and $b_{x}$ and $b_{y}$ denote the amplitude components of the transverse wave propagating in the $z$-direction; $\tau=t \Omega_{\mathrm{i}}$ is the normalized time in units of the inverse ion cyclotron time $\left(\Omega_{\mathrm{i}}\right.$ denotes the non-relativistic ion cyclotron frequency); $\xi=z \Omega_{\mathrm{i}} / V_{\mathrm{A}}$ is a normalized spatial coordinate related to the physical coordinate $z$ ( $V_{\mathrm{A}}$ denotes the Alfvén speed); $N_{1}$ is a positive dimensionless value which, in rigorous fluid theory, is related to the plasma beta $\beta$ through $N_{1}=(1-\beta) / 2$, but in a more accurate kinetic description is a gradually varying function of $\beta$ (Spangler 1991). The factor $\mu$ measures the strength of the plasma wave dispersion effects. The positive dimensionless parameter $\mathrm{N}_{2}$ measures the so-called "non-local" wave effects (Mjølhus \& Wyller 1986, 1988) and is the only term capable of causing wave damping in Eq. (1), because, without the presence of $N_{2}$, the global integral $\int_{-\infty}^{\infty}|\phi(\xi)|^{2} \mathrm{~d} \xi$ is conserved. More details concerning the derivation and the applicability of the Rogister-Mjølhus-Wyller equation are contained in Appendix A.

This paper provides information on several aspects of Eq. (1) which, to our knowledge, have not been previously investigated. The aim is to improve our understanding of the types of responses such non-linear wave models can engender in the interstellar medium. Three aspects of Eq. (1) are considered. First is the question of the spatial and/or temporal structure of solutions to Eq. (1). Second is the question of the energy loss due to the term factored by $N_{2}$. Third is the question of undamped oscillatory solutions to Eq. (1) and their influence on potential acceleration of charged particles. As we shall demonstrate directly, Eq. (1) is exceedingly rich in structural behaviour, so that the patterns of response that can be coaxed out of the equation represent a distinct improvement in our understanding of the physical processes of wave-particle interactions in the interstellar medium.

\section{Some general considerations}

\subsection{Structural forms}

In the search for wave-like behaviour for Eq. (1), the general type of reduction follows the standard path to obtaining a pattern of response. First one considers the response to depend on the combination $\chi$ and $\tau$ where $\chi=\xi-F(\tau)$, with $F(\tau)$ a real function yet to be determined. Then one considers that $\phi(\chi, \tau)$ shall have the dependence

$\phi(\chi, \tau)=A(\tau) B(\chi / H(\tau))$

where $A(\tau), B$ and $H(\tau)$ are functions to be determined. Direct insertion of these considerations into Eq. (1) yields

$\imath H^{2}(\tau) \frac{\mathrm{d} \ln A(\tau)}{\mathrm{d} \tau}-\imath H(\tau) \frac{\mathrm{d} H(\tau)}{\mathrm{d} \tau} \frac{s B_{\mathrm{s}}}{B}-\imath H(\tau) \frac{B_{\mathrm{s}}}{B} \frac{\mathrm{d} F(\tau)}{\mathrm{d} \tau}$

$-\mu \frac{B_{\mathrm{ss}}}{B}+\frac{\imath|A|^{2}}{2} \frac{H(\tau)}{B}$

$\times \frac{\mathrm{d}}{\mathrm{d} s}\left(B\left[N_{1}|B|^{2}+N_{2} \mathrm{P} \int_{-\infty}^{\infty} \frac{\mathrm{d} s_{0}\left|B\left(s_{0}\right)\right|^{2}}{\left(s_{0}-s\right)}\right]\right)=0$

where

$B_{\mathrm{s}} \equiv \frac{\mathrm{d} B}{\mathrm{~d} s} ; \quad B_{\mathrm{ss}} \equiv \frac{\mathrm{d}^{2} B}{\mathrm{~d} s^{2}} ; s \equiv \frac{\chi}{H(\tau)}$.

In order that this separation of variables holds for arbitrary $\tau$ and $s$, it follows from Eq. (3) that one requires

$H^{2}(\tau) \frac{\mathrm{d} \ln A(\tau)}{\mathrm{d} \tau}=c_{0}=$ constant

$H(\tau) \frac{\mathrm{d} H(\tau)}{\mathrm{d} \tau}=c_{1}=$ constant

$H(\tau) \frac{\mathrm{d} F(\tau)}{\mathrm{d} \tau}=c_{2}=$ constant

$H(\tau)|A(\tau)|^{2}=c_{3}=$ constant.

From Eq. (6) it follows that

$H(\tau)=\sqrt{2 c_{1} \tau+h_{0}^{2}}$

where $h_{0}=$ constant, and from Eq. (7)

$F(\tau)=F_{0}+\frac{c_{2}}{c_{1}} H(\tau)$ 
where $F_{0}=$ constant. From Eq. (5) one has

$A(\tau)=A_{0}[H(\tau)]^{c_{0} / c_{1}}=A_{0}\left[2 c_{1} \tau+h_{0}^{2}\right]^{c_{0} / 2 c_{1}}$

where $A_{0}=$ constant.

From the requirement that $F(\tau)$ be real for all $\tau$, it follows that $F_{0}, c_{2}, c_{1}$ and $h_{0}^{2}$ all be real. Further $c_{1}$ must be greater than or equal to zero to avoid $2 c_{1} \tau+h_{0}^{2}$ turning negative, which would drive $F(\tau)$ complex, and thus violate the ansatz. From Eqs. (8) and (11) it then follows that

$c_{3}=\left|A_{0}\right|^{2}$

must be real and positive, and that

$c_{0}+c_{0}^{*}=-c_{1}$.

The imaginary part of $c_{0}$ is not determined by these constraints. (For later use we note that if $c_{1} \rightarrow 0$ then $c_{0}=-\imath \Omega$ with $\Omega$ real, implying that $H \rightarrow h_{0}$ and $F(\tau)=F_{0}+\left(c_{2} \tau / h_{0}\right)$ and that $\left.A(\tau) \rightarrow A_{0} \exp \left(-\imath \Omega \tau / h_{0}^{2}\right).\right)$

With $c_{0}=c_{\mathrm{R}}-\imath \Omega, c_{\mathrm{R}}$ real, Eq. (3) can be written in the general form

$\imath\left(c_{\mathrm{R}}-\imath \Omega\right) B+\left(2 c_{\mathrm{R}} s-c_{2}\right) \imath B_{\mathrm{s}}-\mu B_{\mathrm{ss}}$

$+\frac{\imath\left|A_{0}\right|^{2}}{2} \frac{\mathrm{d}}{\mathrm{d} s}\left(B\left[N_{1}|B|^{2}+N_{2} \mathrm{P} \int_{-\infty}^{\infty} \frac{\mathrm{d} s_{0}\left|B\left(s_{0}\right)\right|^{2}}{\left(s_{0}-s\right)}\right]\right)=0$.

Now note from Eq. (11) that

$|A(\tau)|^{2}=\left|A_{0}\right|^{2}\left[2 c_{1} \tau+h_{0}^{2}\right]^{-1 / 2}$

and that from Eq. (8)

$\int_{-\infty}^{\infty} \mathrm{d} \chi|\phi(\chi, \tau)|^{2}=\int_{-\infty}^{\infty} \mathrm{d} \chi|A(\tau)|^{2}|B(\chi / H(\tau))|^{2}=$

$H(\tau)|A(\tau)|^{2} \int_{-\infty}^{\infty} \mathrm{d} s|B(s)|^{2}=c_{3} \int_{-\infty}^{\infty} \mathrm{d} s|B(s)|^{2}$

so that all spatially bounded solutions of Eq. (14) of the structural form $\phi(\chi, \tau)=A(\tau) B(\chi / H(\tau))$ conserve energy with time for any value of $c_{1}$ not identically zero.

For $c_{1}=0$, however, one has $H \rightarrow h_{0}, c_{\mathrm{R}}=0$ and then one is interested in solutions to

$$
\begin{aligned}
& \Omega B-\imath c_{2} B_{\mathrm{s}}-\mu B_{\mathrm{ss}}+\frac{\imath\left|A_{0}\right|^{2}}{2} \\
& \times \frac{\mathrm{d}}{\mathrm{d} s}\left(B\left[N_{1}|B|^{2}+N_{2} \mathrm{P} \int_{-\infty}^{\infty} \frac{\mathrm{d} s_{0}\left|B\left(s_{0}\right)\right|^{2}}{\left(s_{0}-s\right)}\right]\right)=0 .
\end{aligned}
$$

\subsection{Energy loss and conservative solution}

Spangler (1991) already noted that from Eq. (1) one can derive for the energy change rate

$$
\begin{aligned}
\dot{\epsilon} & \equiv \frac{\mathrm{d}}{\mathrm{d} \tau} \int_{-\infty}^{\infty} \mathrm{d} \xi|\phi(\xi, \tau)|^{2}=-\frac{1}{2} N_{2} \int_{-\infty}^{\infty} \mathrm{d} \xi|\phi(\xi, \tau)|^{2} \\
& \left.\times \frac{\partial}{\partial \xi}\left(P \int_{-\infty}^{\infty} \frac{\mathrm{d} \xi_{0}\left|\phi\left(\xi_{0}, \tau\right)\right|^{2}}{\left(\xi_{0}-\xi\right)}\right]\right)
\end{aligned}
$$

for all solutions of Eq. (1) which are spatially bounded with $|\phi(\xi, \tau)|^{2} \rightarrow 0$ as $|\xi| \rightarrow \infty$ for any finite $\tau$. Thus, if there is to be any energy change then it arises only as a consequence of the nonlocal term factored by $N_{2}$ (Mjølhus \& Wyller 1988). Solutions with $N_{2}=0$ conserve energy. Numerical studies of soliton solutions to Eq. (1) (Flå et al. 1989) found that they damp with time because of the term involving $N_{2}$. So two situations are available: either one has spatially bounded soliton-like solutions that may damp if the right hand side of Eq. (18) is negative, or one has spatially non-bounded solutions (in the sense that $|\phi(\xi, \tau)|^{2}$ does not tend to zero as $\left.|\xi| \rightarrow \infty\right)$, which do not damp. In this subsection we consider the spatially bounded solutions, while Sect. 3 considers the spatially non-bounded solutions.

If $N_{2}$ is small, then the global energy loss rate $\dot{\epsilon}$ is obtained by taking the solutions of Eq. (1) in the absence of the term containing $N_{2}$, i.e. solutions of the standard DNLS, and then using them directly in the right hand side of Eq. (18). According to property (16) only solutions with $c_{1}=0$ are of interest here. We denote solutions to Eq. (17) obtained with $N_{2}=0$ by $D(s)$. Then with $N_{2}=0$,

$\phi\left(s, \tau, N_{2}=0\right)=\phi_{0}(s, \tau)=A_{0} \exp \left(-\imath \Omega \tau / h_{0}^{2}\right) D(s)$

where $D(s)$ satisfies

$\Omega D-\imath c_{2} \frac{\mathrm{d} D}{\mathrm{~d} s}-\mu \frac{\mathrm{d}^{2} D}{\mathrm{~d} s^{2}}+\frac{\imath\left|A_{0}\right|^{2}}{2} \frac{\mathrm{d}}{\mathrm{d} s}\left(D N_{1}|D|^{2}\right)=0$

and the energy loss rate then is approximately

$$
\begin{aligned}
\dot{\epsilon} & =-\frac{1}{2} N_{2}\left|A_{0}\right|^{4} \int_{-\infty}^{\infty} \mathrm{d} s|D(s)|^{2} \\
& \left.\times \frac{\partial}{\partial s}\left(P \int_{-\infty}^{\infty} \frac{\mathrm{d} \xi_{0}\left|D\left(\xi_{0}\right)\right|^{2}}{\left(\xi_{0}-s\right)}\right]\right) .
\end{aligned}
$$

Equation (20) can we rewritten as

$\Omega D+\frac{\imath\left|A_{0}\right|^{2}}{2} \frac{\mathrm{d}}{\mathrm{d} s}\left(D\left[N_{1}|D|^{2}-\frac{2 c_{2}}{\left|A_{0}\right|^{2}}\right]\right)-\mu \frac{\mathrm{d}^{2} D}{\mathrm{~d} s^{2}}=0$.

The general solution to Eq. (22) is not difficult to obtain but is somewhat involved. First re-write variables with

$D=R \exp (\imath \psi)$

where $R$ and $\psi$ are both real. Then Eq. (22) takes the form

$$
\begin{aligned}
& \Omega R-\frac{\left|A_{0}\right|^{2}}{2} \frac{\mathrm{d} \psi}{\mathrm{d} s}\left(R\left[N_{1} R^{2}-\frac{2 c_{2}}{\left|A_{0}\right|^{2}}\right]\right) \\
& +\frac{\imath\left|A_{0}\right|^{2}}{2} \frac{\mathrm{d}}{\mathrm{d} s}\left(R\left[N_{1} R^{2}-\frac{2 c_{2}}{\left|A_{0}\right|^{2}}\right]\right) \\
& -\mu\left[\frac{\mathrm{d}^{2} R}{\mathrm{~d} s^{2}}+2 \imath \frac{\mathrm{d} \psi}{\mathrm{d} s} \frac{\mathrm{d} R}{\mathrm{~d} s}-R\left(\frac{\mathrm{d} \psi}{\mathrm{d} s}\right)^{2}+\imath R \frac{\mathrm{d}^{2} \psi}{\mathrm{d} s^{2}}\right]=0 .
\end{aligned}
$$

Collecting real and imaginary parts separately we obtain

$$
\begin{gathered}
\Omega R-\frac{\left|A_{0}\right|^{2}}{2} \frac{\mathrm{d} \psi}{\mathrm{d} s}\left(R\left[N_{1} R^{2}-\frac{2 c_{2}}{\left|A_{0}\right|^{2}}\right]\right) \\
-\mu\left[\frac{\mathrm{d}^{2} R}{\mathrm{~d} s^{2}}-R\left(\frac{\mathrm{d} \psi}{\mathrm{d} s}\right)^{2}\right]=0
\end{gathered}
$$


and

$$
\begin{gathered}
+\frac{\left|A_{0}\right|^{2}}{2} \frac{\mathrm{d}}{\mathrm{d} s}\left(R\left[N_{1} R^{2}-\frac{2 c_{2}}{\left|A_{0}\right|^{2}}\right]\right) \\
-\mu\left[2 \frac{\mathrm{d} \psi}{\mathrm{d} s} \frac{\mathrm{d} R}{\mathrm{~d} s}+R \frac{\mathrm{d}^{2} \psi}{\mathrm{d} s^{2}}\right]=0 .
\end{gathered}
$$

Note that both Eqs. (25) and (26) involve only $\frac{\mathrm{d} \psi}{\mathrm{d} s}$ and $\frac{\mathrm{d}^{2} \psi}{\mathrm{d} s^{2}}$ but not $\psi$ explicitly. Then set $M=\frac{\mathrm{d} \psi}{\mathrm{d} s}$ when Eq. (26) takes the form (using the abbreviation $R_{\mathrm{s}}=\frac{\mathrm{d} R}{\mathrm{~d} s}$ etc.)

$\mu\left[R M_{\mathrm{s}}+2 M R_{\mathrm{s}}\right]=\frac{\left|A_{0}\right|^{2}}{2} \frac{\mathrm{d}}{\mathrm{d} s}\left(R\left[N_{1} R^{2}-\frac{2 c_{2}}{\left|A_{0}\right|^{2}}\right]\right)$

which has an immediate first integral

$$
\begin{aligned}
M R^{2}= & \frac{\left|A_{0}\right|^{2}}{2 \mu} \int^{s} \mathrm{~d} s_{1} R\left(s_{1}\right) \\
& \times \frac{\mathrm{d}}{\mathrm{d} s_{1}}\left(R\left(s_{1}\right)\left[N_{1} R\left(s_{1}\right)^{2}-\frac{2 c_{2}}{\left|A_{0}\right|^{2}}\right]\right)
\end{aligned}
$$

or

$$
M=\frac{\left|A_{0}\right|^{2}}{2 \mu}\left(\frac{1}{4} N_{1} R^{2}-\frac{c_{2}}{\left|A_{0}\right|^{2}}+\frac{\Lambda_{1}}{R^{2}}\right)=\frac{\mathrm{d} \psi}{\mathrm{d} s}
$$

where $\Lambda_{1}$ is the constant of integration.

Using the solution (29) in Eq. (25) yields

$\Omega R-\frac{\left|A_{0}\right|^{4}}{4 \mu} R\left[N_{1} R^{2}-\frac{2 c_{2}}{\left|A_{0}\right|^{2}}\right]\left(\frac{1}{4} N_{1} R^{2}-\frac{c_{2}}{\left|A_{0}\right|^{2}}+\frac{\Lambda_{1}}{R^{2}}\right)$

$-\mu \frac{\mathrm{d}^{2} R}{\mathrm{~d} s^{2}}+\frac{\left|A_{0}\right|^{4}}{4 \mu} R\left(\frac{1}{4} N_{1} R^{2}-\frac{c_{2}}{\left|A_{0}\right|^{2}}+\frac{\Lambda_{1}}{R^{2}}\right)^{2}=0$.

Equation (30) is of the generic form

$\frac{\mathrm{d}^{2} R}{\mathrm{~d} s^{2}}=F(R)$

where

$$
\begin{aligned}
F(R)= & \frac{\Omega}{\mu} R+\frac{\left|A_{0}\right|^{4}}{4 \mu} R\left(\frac{1}{4} N_{1} R^{2}-\frac{c_{2}}{\left|A_{0}\right|^{2}}+\frac{\Lambda_{1}}{R^{2}}\right) \\
& \times\left(\frac{c_{2}}{\left|A_{0}\right|^{2}}+\frac{\Lambda_{1}}{R^{2}}-\frac{3}{4} N_{1} R^{2}\right) .
\end{aligned}
$$

The general method of solving Eq. (31) is to write

$\frac{\mathrm{d} R}{\mathrm{~d} s}=R W^{1 / 2}$

when

$$
\begin{aligned}
\frac{\mathrm{d}^{2} R}{\mathrm{~d} s^{2}} & =W^{1 / 2} \frac{\mathrm{d} R}{\mathrm{~d} s}+\frac{R}{2} W^{-1 / 2} \frac{\mathrm{d} W}{\mathrm{~d} s}=W R+\frac{R^{2}}{2} \frac{\mathrm{d} W}{\mathrm{~d} R} \\
& =\frac{1}{2} \frac{\mathrm{d}}{\mathrm{d} R}\left(W R^{2}\right) .
\end{aligned}
$$

Hence Eq. (31) yields

$W R^{2}=2 \int^{R} F\left(R_{1}\right) \mathrm{d} R_{1}$ so that

$\frac{\mathrm{d} R}{\mathrm{~d} s}=\left[2 \int^{R} F\left(R_{1}\right) \mathrm{d} R_{1}\right]^{1 / 2}$

and so

$s=\int^{R} \frac{\mathrm{d} R_{1}}{\left[2 \int^{R_{1}} F\left(R_{2}\right) \mathrm{d} R_{2}\right]^{1 / 2}}$.

From Eq. (29) one has

$\frac{\mathrm{d} \psi}{\mathrm{d} s}=M(R)$

yielding

$\frac{\mathrm{d} \psi}{\mathrm{d} R} \frac{\mathrm{d} R}{\mathrm{~d} s}=M(R)$.

Hence

$\psi=\int^{R} \frac{\mathrm{d} R_{1} M\left(R_{1}\right)}{\left[2 \int^{R_{1}} F\left(R_{2}\right) \mathrm{d} R_{2}\right]^{1 / 2}}$.

The general problem has, therefore, been reduced to simple quadratures whose evaluation depends explicitly on the conditions chosen to determine the constants of integration. Hence, all spatially bounded structural forms with $\phi_{0}(s, \tau)=A_{0} \exp \left(-\imath \Omega t / h_{0}^{2}\right) D(s)$ have been obtained.

\section{Exact wave solutions}

Apart from the spatially bound solutions to Eq. (17), there are also wave solutions which are of interest in their own right. This interest stems from the fact that if, indeed, all soliton-like solutions eventually decay (for $N_{2} \neq 0$ ), then only the wave-like solutions persist. These wave solutions may, in turn, be able to preferentially accelerate charged particles (see Sect. 4).

To obtain wave solutions to Eq. (17) is relatively easy. Set

$B(s)=\exp (\imath k s)$

Then, remembering $\phi=A_{0} \exp \left(i k s-\imath \Omega h_{\mathrm{o}}^{-2} \tau\right)$, inserting Eq. (41) into Eq. (17) gives

$\Omega=-\mu k^{2}-c_{2} k+\frac{N_{1}\left|A_{0}\right|^{2}}{2} k$

for all values of $N_{2}$. Thus the waves are totally uninfluenced by the appearance of the energy decay term factored by $N_{2}$. Rewrite the wave as $\phi=A_{0} \exp (i k s-\imath \omega \tau)$, with $\omega=\Omega+c_{2} k$, one has the dispersion relation

$\omega=k\left(\frac{N_{1}\left|A_{0}\right|^{2}}{2}-\mu k\right)$.

The waves are forward propagating if

$k_{*} \equiv \frac{N_{1}\left|A_{0}\right|^{2}}{2 \mu}>k$ 
and reverse propagating otherwise. Thus long wavelengths $\left(k_{*}>k\right)$ propagate in the positive sense, while short wavelengths $\left(k>k_{*}\right)$ propagate in the negative sense. The phase velocity, $\omega / k=v_{\mathrm{p}}$, is

$v_{\mathrm{p}}=\mu\left(k_{*}-k\right)$

and the group velocity is

$v_{\mathrm{g}}=\frac{\partial \omega}{\partial k}=\mu\left(k_{*}-2 k\right)$.

For positive sense waves one has $v_{\mathrm{p}}>v_{\mathrm{g}}$ and $v_{\mathrm{g}}$ is oppositely directed to $v_{\mathrm{p}}$ for $k_{*}>k>k_{*} / 2$. For negative sense waves the opposite is true: $\left|v_{\mathrm{g}}\right|>\left|v_{\mathrm{p}}\right|$, but both are always directed in the same sense.

This difference in propagation character at short and long wavelengths, dependent on the amplitude of the wave (through $k_{*}=\frac{N_{1}\left|A_{0}\right|^{2}}{2 \mu}$ ), would suggest that there should be at least one choice of $k$ that could lead to particle acceleration. This part of the problem is now considered.

\section{Charged particle acceleration}

The wave solutions for the magnetic field, $b_{x}+\imath b_{y}$, obey

$$
\begin{aligned}
b_{x}+\imath b_{y} & =B_{0} \phi=B_{0}\left|A_{0}\right| \mathrm{e}^{\imath \rho} \\
& =\left|A_{0}\right| B_{0} \mathrm{e}^{\imath \eta} \exp \left(i k z \Omega_{\mathrm{i}} / V_{\mathrm{A}}-\imath \omega \Omega_{\mathrm{i}} t\right)
\end{aligned}
$$

where we have restored the dimensionality. Here $\eta$ is the relative phase on $z=0=t$ between $b_{x}$ and $b_{y}$; the relation between the dimensionless $\omega$ and $k$ is given by the dispersion relation (43). Correspondingly, for the associated real electric field, $\boldsymbol{E}=E_{x} \boldsymbol{e}_{x}+E_{y} \boldsymbol{e}_{y}$, one has

$E_{x}+\imath E_{y}=-\imath \frac{V_{\mathrm{A}}}{c} \frac{\omega}{k}\left(b_{x}+\imath b_{y}\right)$.

For a particle of rest mass $m$, charge $e$ under the action of the electric and magnetic fields given by Eqs. (47) and (48), one has the equations of motion as

$\frac{\mathrm{d} p_{x}}{\mathrm{~d} t}=-\frac{e}{c}\left[v_{z}-\frac{\omega V_{\mathrm{A}}}{k}\right]\left|A_{0}\right| B_{0} \sin (\rho(z, t))+\frac{e v_{y} B_{0}}{c}$

$\frac{\mathrm{d} p_{y}}{\mathrm{~d} t}=\frac{e}{c}\left[v_{z}-\frac{\omega V_{\mathrm{A}}}{k}\right]\left|A_{0}\right| B_{0} \cos (\rho(z, t))-\frac{e v_{x} B_{0}}{c}$

$\frac{\mathrm{d} p_{z}}{\mathrm{~d} t}=\frac{e\left|A_{0}\right| B_{0}}{c}\left[v_{x} \sin (\rho(z, t))-v_{y} \cos (\rho(z, t))\right]$

with

$\rho(z, t)=k z \Omega_{\mathrm{i}} / V_{\mathrm{A}}-\omega \Omega_{\mathrm{i}} t+\eta$

in terms of the momentum $\boldsymbol{p}$ and with the connection $m \boldsymbol{v}=\boldsymbol{p}\left[1+\left(p^{2} / m^{2} c^{2}\right)\right]^{-1 / 2}$. Moreover one has

$\frac{\mathrm{d} x}{\mathrm{~d} t}=v_{x}, \quad \frac{\mathrm{d} y}{\mathrm{~d} t}=v_{y}, \quad \frac{\mathrm{d} z}{\mathrm{~d} t}=v_{z}$.

Multiplying Eq. (49) with $\cos \rho$, Eq. (50) with $\sin \rho$, adding the results and using Eq. (51) yields the relation

$\frac{1}{\left|A_{0}\right|} \frac{\mathrm{d} p_{z}}{\mathrm{~d} t}+\cos \rho \frac{\mathrm{d} p_{x}}{\mathrm{~d} t}+\sin \rho \frac{\mathrm{d} p_{y}}{\mathrm{~d} t}=0$.
Also, multiplying Eq. (49) with $m \gamma p_{x}$, Eq. (50) with $m \gamma p_{y}$, where

$\gamma=\sqrt{1+\frac{p^{2}}{m^{2} c^{2}}}$

and adding the results yields

$\frac{\mathrm{d} p^{2}}{\mathrm{~d} t}=\frac{2 \omega V_{\mathrm{A}} m \gamma}{k} \frac{\mathrm{d} p_{z}}{\mathrm{~d} t}$

where we use Eq. (51). Equation (56) has the complete integral

$m c \sqrt{1+\frac{p^{2}}{m^{2} c^{2}}}-\frac{\omega V_{\mathrm{A}}}{k c} p_{z}=$ const.

which can be written as

$\gamma\left[1-\frac{\omega V_{\mathrm{A}} v_{z}}{k c^{2}}\right]=$ const.

Consider particles starting at $z=0=t$ with velocity components $v_{x 0}, v_{y 0}, v_{z 0}$. At some later time, let the $z$ component of velocity have reached the value $v_{z \mathrm{f}}$. The corresponding value of $\rho(z, t)$ is

$\rho(z, t)=\eta+t\left[k v_{z \mathrm{f}} \frac{\Omega_{\mathrm{i}}}{V_{\mathrm{A}}}-\omega \Omega_{\mathrm{i}}\right]$.

This value will be independent of time if

$v_{z \mathrm{f}}=\omega V_{\mathrm{A}} / k$.

Then, Eqs. (49)-(50) simply solve as

$v_{x}(t)=v_{\perp} \cos (\Omega t) ; \quad v_{y}(t)=v_{\perp} \sin (\Omega t) \quad \Omega=\frac{e B_{0}}{m c \gamma}$

and Eq. (58) yields for the relation of final (f) and initial (i) values

$\frac{\gamma_{\mathrm{f}}}{\gamma_{\mathrm{i}}}=\frac{1-\frac{\omega V_{\mathrm{A}} v_{z 0}}{k c^{2}}}{1-\frac{V_{\mathrm{A}}^{2} \omega^{2}}{k^{2} c^{2}}}$

which is equivalent to

$$
\begin{aligned}
{\left[m^{2} c^{2}+p_{\perp}^{2}+\right.} & \left.p_{z 0}^{2}\right]\left[1-\frac{\omega V_{\mathrm{A}} v_{z 0}}{k c^{2}}\right]^{2}= \\
& {\left[m^{2} c^{2}+p_{\perp}^{2}+p_{z \mathrm{f}}^{2}\right]\left[1-\frac{V_{\mathrm{A}}^{2} \omega^{2}}{k^{2} c^{2}}\right]^{2} . }
\end{aligned}
$$

Hence: if the initial particle velocity $v_{z 0}$ in the $z$-direction is chosen smaller than $V_{\mathrm{A}} \omega / k$ then the final energy is larger than the initial energy, i.e. particles are accelerated.

The condition for acceleration $v_{z 0}<\frac{V_{\mathrm{A}} \omega}{k}=v_{\mathrm{p}}$ can be written with Eq. (45) as

$v_{z 0}<\mu\left(k_{*}-k\right)$

it follows that acceleration can occur at least when

$\mu\left(k_{*}-k\right)>c$.

Considerations of particle motion other than that given by Eq. (60) may also provide selective velocity ranges for particle acceleration. However, it is sufficient to show here that at least some particles are accelerated by the wave solutions of the nonlinear Landau damping equation. 


\section{Discussion and conclusions}

The purpose of this paper has been to develop further some of the rich structure of properties associated with the wave kinetic equation for non-linear waves. Three aspects of the equation properties have been considered:

(1) First, some of the basic properties of spatially bounded soliton-like solutions were provided indicating how such structures can develop.

(2) Second, particular structured forms, spatially bounded, were reduced to simple quadratures, thereby providing general analytic solutions in quadrature form. The patterns of behaviour for such soliton-like solutions were shown to be dependent on the initial and/or boundary conditions one chooses to impose, leading to a rich diversity of possible behaviors.

(3) Third, a class of wave-like solutions were derived that do not damp with time, independent of the value for the so-called "non-local" term in the equation that controls energy loss. These wave-like solutions were shown to be capable of accelerating charged particles. It may be that more general conditions for particle acceleration can be found than those developed here. However, the fact that some particles, at the least, can reach higher energies using the waves, suggests that a more general investigation of the spatially bounded soliton-like behaviours should also provide acceleration capabilities. The complexity and diversity of possible structural soliton patterns has so far precluded a detailed investigation of the particle acceleration capabilities of all classes of solutions to the non-linear Landau damping wave kinetic equation. But that one class of solutions does provide for such acceleration augers well for any further investigation involving more general classes of solutions.

The main point is that the influence of non-linear effects on wave characteristics for energy gain and/or loss in the interstellar medium is not to be lightly dismissed. In particular, for heating of the interstellar medium, the investigation in Sect. 2.2 shows that the energy transfer rate (as approximated by Eq. (21)) can be evaluated in terms of quadratures dependent on the initial and/or boundary conditions imposed. This use of exact solutions to the DNLS equation is a major improvement over the ad hoc suggestions (Spangler 1991) for the structured solutions. The ad hoc behaviors do not come even close to satisfying the basic equations under consideration by direct insertion into the equations. For the classes of solutions described by decaying soliton behaviors, the influence of such heating (cooling) events can seriously affect the understanding of the balance of wave heating and radiative cooling in the interstellar medium. The basic solutions exhibited here would, seemingly, be a significant step forward in our long-term attempts to understand this problem.

Equally, the presence of oscillatory solutions, independent of $N_{2}$ for any and all values of $N_{2}$, would seem to indicate that such waves play a role in the long-term evolution of the interstellar medium. If the soliton-like structures decay (for $N_{2} \neq 0$ ) then one is eventually left with a body of non-linear oscillating waves. These waves propagate in both positive and negative senses (depending on their wavenumbers), suggesting a bifurcation of energy flux directions at large and small spatial scales. Such a division is of significance not only for the long term behavior of the interstellar medium, but also for particle energization. This paper has shown how all of these problems can be addressed using the one-dimensional non-local DNLS equation. It is clear that the richness of patterns can only increase with multi-dimensional equations of similar sort.

The total effect of all such possibilities on the long term evolution of the interstellar medium is seen but murkily at present, but also represents an exciting challenge. The soliton behaviors, the forward and reverse propagating oscillatory waves, and the particle acceleration by the nonlinear waves, all provide processes that are fundamentally beholden to the non-linear terms, without which all such effects would vanish. And these non-linear processes form the start of a significant combined integration of information in our attempts to understand the intertwined evolution of waves and charged particles in the interstellar medium.

Acknowledgements. We are grateful to Drs. M. Goossens and Y. Voitenko for pointing out to us the underlying assumptions in the derivation of the Rogister-Mjølhus-Wyller equation. We gratefully acknowledge support by the Deutsche Forschungsgemeinschaft through Sonderforschungsbereich 191.

\section{Appendix A: Applicability of the Rogister- Mjølhus-Wyller equation to interstellar turbulence}

The Rogister-Mjølhus-Wyller Eq. (1) is a "Kinetically Modified Derivative Nonlinear Schrödinger equation" (KMDNLS) where the modification arises from the nonlocal term scaling with $N_{2}$. It reduces to the Derivative Nonlinear Schrödinger equation (DNLS) when $N_{2}=0$.

The derivation of the DNLS equation has been reviewed by Dawson \& Fontan (1990) and Mjølhus \& Wyller (1986). It has been originally obtained from the set of nondissipative MHD equations, where all variables are supposed to depend only on the z-coordinate (Sakai \& Sonnerup 1983; Spangler \& Sheerin 1982) by means of a reductive perturbation method (Taniui \& Wei 1968). Due to this geometric restriction, the model obtained is unidirectional and $B_{z}=B_{0}$ always, so that longitudinal perturbations of the magnetic field cannot be described. The complex variable $b=\phi B_{0}$ represents the real valued transverse field components $B_{x, y}$ according to

$b=\epsilon^{-1 / 2}\left[B_{x}+\imath B_{y}\right]$

and the profile and evolution coordinates $\xi$ and $\tau$ are stretched according to

$\xi=\epsilon\left( \pm z-V_{\mathrm{A}} t\right), \quad \tau=\epsilon^{2} t$. 
The small parameter $\epsilon$ characterizes the simultaneous weak nonlinearity and weak dispersion (i.e. long wave length) requiring that the characteristic scale of turbulence $l$ fulfills

$\frac{V_{\mathrm{A}}}{l \Omega_{\mathrm{i}}}=O(\epsilon), \epsilon \ll 1$

which is equivalent to the low frequency limit $\omega / \Omega_{\mathrm{i}}=$ $O(\epsilon)$. The smallness of $\epsilon$ also imples that the magnetic field perturbation is $\epsilon^{-1 / 2}$ larger than the density fluctuation. As long as we apply the DNLS equation to parallel propagating Alfven waves at frequencies much less than the proton gyrofrequency, both limiting restrictions are appropriate.

Rogister (1971) was the first in deriving the DNLS from the full three dimensional quasi-neutral Vlasov equations together with the pre-Maxwell's equations (without the displacement current). A perturbation expansion equivalent to the reductive perturbation method led to an equation containing all the terms of the DNLS, but also with the additional non-local term representing resonant particles. Mjølhus \& Wyller (1988) started from the guiding center plasma model to infer the KMDNLS Eq. (1). At least two assumptions are hidden in the derivation of the KNDLS equation that can make the applicability of the KNDLS equation to nonlinear MHD waves in the interstellar medium less than optimal:

(1) The assumption that the velocity distribution of resonant particles is constant in time leads to the dissipative coefficient $N_{2}$ in Eq. (1) being independent of time. If the velocity distribution of the resonant particles, which causes the wave-particle interaction, evolves much faster than the wave amplitudes then the quasilinear diffusion smoothes out the velocity distribution, thus quenching the Landau resonance and the coefficient $N_{2}$. Then one again would have the DNLS Eq. (1) with $N_{2}=0$.

(2) The assumption of time reversibility of Landau damping, which gives rise to the Hilbert transform operator in the nonlocal term of Eq. (1), might be violated. Such an effect can happen because of a change of the dissipative term due to scattering of particles at small angles.
A non-local term would persist but would be more complexly described than by a Hilbert transform.

Both assumptions become especially crucial for the asymptotic states of the turbulence at long times.

Unfortunately, compared to the interplanetary medium, where in-situ measurements on spacecrafts of the turbulent properties of the interplanetary medium are available, the observational data for interstellar medium turbulence is scarce, and, as mentioned already, is mainly based on line-of-sight electron density fluctuation measurements through a multi-phase interstellar medium from scintillation, dispersion measure and Faraday rotation measure observations. Therefore no direct observational clue on the validity of the underlying assumptions (1) and (2) in the interstellar medium can be provided. It is, however, important to keep these hidden assumptions in mind when applying the solution properties of the KNDLS equation presented in this work to the interstellar medium.

We finally mention that Dawson \& Fontan (1990) have investigated the soliton-behaviour of Eq. (1) with $N_{2}=0$, whereas Flå et al. (1989) found numerically that with $N_{2} \neq 0$ the soliton-like modes damp with time.

\section{References}

Armstrong, J. W., Rickett, B. J., \& Spangler, S. R. 1995, ApJ, 443, 209

Dawson, S. P., \& Fontan, C. F. 1990, ApJ, 348, 761

Flå, A. L., Mjølhus, E., \& Wyller, J. 1989, Phys. Scr., 40, 219

Lerche, I., \& Schlickeiser, R. 2001, A\&A, 366, 1008

Mjølhus, E., \& Wyller, J. 1986, Phys. Scr., 33, 442

Mjølhus, E., \& Wyller, J. 1988, J. Plasma Phys., 40, 299

Rogister, A. 1971, Phys. Fluids, 14, 2733

Sakai, J., \& Sommerup, B. U. Ö. 1983, JGR, 88, 9069

Spangler, S. R. 1990, Phys. Fluids, B2, 408

Spangler, S. R. 1991, ApJ, 376, 540

Spangler, S. R. 1999, in Plasma Turbulence and Energetic Particles in Astrophysics, ed. M. Ostrowski, \& R. Schlickeiser, Krakow, ISBN 83-908592-0-3, 1

Spangler, S. R., \& Sheerin, J. P. 1982, J. Plasma Phys., 27, 193

Taniuti, T., \& Wei, C. 1968, J. Phys. Soc. Jpn., 24, 941 